ORIGINAL ARTICLE

\title{
Effect of vestibular rehabilitation therapy on visual and motor functions in children with dyspraxia
}

\author{
MAHA ABD ELLATIF ${ }^{1 *}$, KAMAL E.SHOUKRY², GEHAN M. ABD EL MAKSOUD², AHMED E. CHEDID³, AHMED F. GENEDY ${ }^{4}$ \\ ${ }^{1}$ Physical therapist at Wadi Elneel Hospital, Cairo, Egypt. \\ ${ }^{2}$ Professor of Physical therapy, Department of Pediatric Physical Therapy, \\ Faculty of Physical Therapy, Cairo University, Giza, Egypt. \\ ${ }^{3}$ Assistant professor Audio-Vestibular Medicine, The Military medical academy, Egypt. \\ ${ }^{4}$ Professor of physical medicine and rehabilitation, The Military Medical Academy, Egypt. \\ ${ }^{\star}$ Corresponding author: Maha Abd Ellatif, Email : Mahaaaa2007@gmail.com, Tel : +20 1020889874
}

\begin{abstract}
Purpose: To investigate the effect of vestibular rehabilitation therapy program on the visual and motor functions in children with dyspraxia.

Method: Twelve children with dyspraxia from both genders, aged from 4 to 12 years, are included in this study. All of them had movement dyspraxia affecting their ability to stand and walk alone according to chick list of developmental dyspraxia. Also, they had level 2 or 3 of visual function level. They received vestibular rehabilitation therapy program in addition to traditional physical therapy program, for three times/ week, for three months. Visual and gross motor functions were assessed before and after three months of treatment using Hoyt's levels of visual function and Peabody Developmental Motor Scale (PDMS- 2) respectively.

Results: Results showed highly significant improvement in visual function level and gross motor functions for the study group when comparing it's results before and after three months of treatment $(P \leq 0.05)$

Conclusion: Vestibular rehabilitation therapy program is an effective modality for improving visual and motor functions in children with dyspraxia.

Key words: Dyspraxia, motor functions, Vestibular Rehabilitation Therapy program, visual functions.
\end{abstract}

\section{INTRODUCTION}

Dyspraxia is a developmental disability of organization of movement. It is caused by an immaturity of the brain resulting in messages not being properly transmitted to the body. It's subtype of Developmental Co-ordination Disorder (DCD) and it's considered as specific disorder in which an individual presents with deficits in conceptualization, planning and execution of movement [1].

Most individuals with dyspraxia manifest a combination of both ideational or planning dyspraxia and ideomotor or executive dyspraxia. Ideational or planning dyspraxia affects the planning and coordination, and ideomotor or executive dyspraxia affects the fluency and speed of motor activities ${ }^{[2]}$.

Dyspraxia is a neurological disorder that impacts an individual's ability to plan and process motor tasks. Individuals with dyspraxia often have language problems, and sometimes a degree of difficulty with thought and perception. Dyspraxia, however, does not affect the person's intelligence, although it can cause learning problems in children. Developmental dyspraxia is an immaturity of the organization of movement. The brain does not process information in a way that allows for a full transmission of neural messages. A person with dyspraxia finds it difficult to plan what to do, and how to do it. The National Institute of Neurological Disorders and Stroke (NINDS) describe people with dyspraxia as being "out of sync" with their environment ${ }^{[3]}$.

The vestibular system is a sensory system that is responsible for providing our brain with information about motion, head position, and spatial orientation; it is also involved with motor functions that allow us to keep our balance, stabilize our head and body during movement, and maintain posture. Thus, the vestibular system is for normal movement and equilibrium ${ }^{[4]}$.

The vestibular system's main function is to sense head movements, especially involuntary ones, and counter them with reflexive eye movements and postural adjustments that keep the visual world stable and keep us from falling ${ }^{[5]}$.

Although vestibular and postural control deficits have been documented in adults with central nervous system involvement, similar investigations of children are scarce. It's logical to assume that like adults, children with traumatic brain injury or other central CNS conditions present with similar vestibular deficits ${ }^{[6]}$. Children whose vestibular dysfunction results in poor muscle coordination (or dyspraxia) may not develop the postural responses necessary to keep upright. They may exhibit awkward, uncoordinated and clumsy moves, often falling, bumping into furniture and losing their balance. Such conditions may be further compounded by visual problems caused by their vestibular dysfunction. As eye movements are influenced by the vestibular system, these children may lack adequate gaze stability and the ability to focus on moving objects while staying still (or vice versa). Lastly, a vestibular disorder may also interfere with their social relationships, for they find it hard to manage personal space in terms of gauging their physical proximity to others, or judging where people are, especially in a crowd. Professionals who treat dyspraxia strive to improve the child's ability to integrate sensory integration from the tactile, vestibular and proprioceptive senses. Children who can integrate input from these senses are more adaptable and ready for learning ${ }^{[7]}$.

Vestibular Rehabilitation Therapy (VRT) is an exercisebased program designed to promote CNS compensation for inner ear deficits. The goal of VRT is to retrain the brain to recognize and process signals from the vestibular system in coordination with vision and proprioception [8]. Moreover, vestibular rehabilitation (VR) or vestibular rehabilitation therapy is a specialized form of therapy intended to alleviate both the primary and secondary problems caused by vestibular disorders. It is an exercise-based program primarily designed to reduce vertigo and dizziness, gaze instability, and/or imbalance and falls ${ }^{[9]}$.

Children with vestibular disorders often respond well to a specialized form of therapy called vestibular rehabilitation therapy. VRT is an exercise program tailored to address eye-movement control, dynamic visual acuity, balance, developmental reflexes, and body-movement functions. VRT exercises should be based on a child's age, interests, level of comprehension, and test results ${ }^{[10]}$.

Vestibular disturbances are under diagnosed in children. However, balance impairment may compromise the normal development of affected children. The appropriate therapeutic approach has not been agreed on for this age group. Vestibular rehabilitation therapy has excellent results in adults, but very few data exist regarding its results in children ${ }^{[11]}$. Therefore, the aim of this study was to investigate the effect of VRT program on the visual and motor functions in children with dyspraxia. 


\section{MATERIALS AND METHODS}

Study Design: This pretest- posttest quasi experimental study was conducted at the outpatient clinic of Faculty of Physical Therapy - Cairo University during the period from September 2019 to February 2020.

Ethics: It has been approved by Ethical Committee of Research of the Faculty of Physical Therapy- Cairo University with No: PT. REC 021/ 00106. Informed consent form was submitted from parents of each child.

Study population: Children with dyspraxia from both sexes, aged from $4-12$ years of age, were participated in this study. They had movement dyspraxia affecting their ability to stand alone and walk according to chick list of developmental dyspraxia. They had limitations in some of their visual functions (Visual focusing and visual tracking) which had been assessed by Hoyt's levels of visual function. Children were excluded from the study if they had seizures and/ or had IQ below 70 .

The study group received VRT program for $30 \mathrm{~min}$./ session, three times/ week for three months in addition to traditional physical therapy program.

Sample size calculation: Sample size calculation was done using the comparison of standard score of locomotion subtest of PDMS 2 between before and after our intervention, as it was the primary outcome of our study. Searching the literature failed to find any previous results that can be used to build up sample size, therefore, we performed a pilot study to get usable results. As reported in our pilot study, the mean \pm SD of standard score before the intervention was approximately $1.2 \pm 0.4$, while after the intervention, it was approximately $2.3 \pm 0.9$. Accordingly, we calculated that the minimum proper sample size was 12 cases to be able to detect a real difference of one unit of the standard score with $95 \%$ power at $\alpha=0.05$ level using Paired t test for dependent samples. Sample size calculation was done using Stats Direct statistical software version 2.7.2 for MS Windows, Stats Direct Ltd., Cheshire, UK.

Therefore, twelve children with dyspraxia of both sexes participated in this study. They selected from outpatient clinic of Faculty of Physical Therapy / Cairo University.

\section{Procedures}

\section{a- Evaluative procedures:}

1- Assessment of dyspraxia

A chick list of developmental dyspraxia was used to evaluate all children before starting the study. The following is a list of some tasks and the ages by which almost of all children have successfully motor planned those tasks. A normal child usually performs most of these tasks at ages earlier than those listed. If the child appears to think and reason all right, but has the trouble learning many of these skills by the ages listed, he may be dyspraxic. It is important to take in account circumstances that may have given a particular child more or less opportunity to learn certain task ${ }^{[12]}$.

2- Assessment of visual function level

Hoyt's level of visual function was used to determine the level of visual function for children participated in this study.

3- Assessment of gross motor function

The Peabody Developmental Motor Scale was used to evaluate the gross motor skills level of children participating in this study. It provides a comprehensive sequence of gross and fine motor skills, from which the therapist can determine the relative developmental skills level of a child, identify the skills that are not completely developed and plan an instructional program to develop those skills. This scale is standardized, reliable and valid assessment tool that allows meeting the needs of various users. It may be translated into specific instructional program ${ }^{[13]}$. Locomotion subtest of PDMS was assessed for all children in this study. The standard score and age equivalent of locomotion subtest were calculated in this study.

\section{b- Treatment procedures}

All children participated in this study received the following vestibular rehabilitation therapy in addition to traditional physical therapy program. The treatment program was applied for one hour and half, three sessions per week, for three successive months.

\section{A-Vestibular Rehabilitation Therapy Program (VRT)}

The following exercises were applied for all children in this study for $30 \mathrm{~min} / \mathrm{session}, 3$ times/ week for 3 months.

\section{A- Eye movement exercises:}

\section{a- Gaze:}

- Exercise 1:

In complete dark room, the child was encouraged to focus on the toy. The light toy was turned on for 30 seconds then turned off for 5 seconds. This exercise was repeated at midline then at right and left directions for 5- 10 times for each direction.

\section{- Exercise 2:}

While using black light, therapist introduced black and white images in midline in front of child's face at distance equal to/ less than the child's arm length, encouraging him/ her to keep eyes focused on the pictures for $20 \mathrm{sec}$. Different pictures were used to maintain child's focus and interest.

\section{- Exercise 3:}

While using black lights, therapist introduced the animal roll in front of child's face at distance of one meter in mid line. Therapist introduced each picture for 20-30 seconds then, changed the pictures while telling story.

\section{b- Saccade:}

\section{- Exercise 1:}

In completely dark room, the therapist placed torch/ light toy in front of child's face at distance of $10 \mathrm{~cm}$. the torch/ light toy was turned on for $30 \mathrm{sec}$., then turned off for $5 \mathrm{sec}$. The therapist changed the position of torch/ light toy placing it in up, down, right and left directions. Child was encouraged to follow the movable objects with his/ her eyes while maintaining the head in static position.

\section{- Exercise 2:}

In completely dark room, the torch's light was directed toward the wall, while the child sat one meter away from the wall. The therapist turned the torch on for 30 seconds and moved its light up and down while encouraged the child to track the light spot on the wall, then turned off the torch for 5 seconds. The previous procedure was repeated, but therapist moved the torch's light to right and left directions on the wall.

\section{c- Pursuit eye movement with balance training:}

\section{- Exercise 1:}

In completely dark room, therapist used disco lights and encouraging the child to follow the movable light spots with eyes only, while the child's head stable (static position). The therapist maintained the head in static position for 2 minutes at mid line, then the therapist moved child's head slowly to right and left directions and maintained child's head in static position at each direction for 2 minutes encouraging the child to follow the movable light spots with eyes only. This exercise repeated 3-5 times for each direction.

\section{- Exercise 2:}

While using disco lights in completely dark room, the therapist encouraged the child to follow the movable light spots while bouncing him/ her up and down. This exercise was repeated 3-5 times while bouncing the child for 2 minutes at each time.

\section{- Exercise 3:}

While using disco lights in completely dark room, the therapist encouraged the child to follow the movable light spots while using several surfaces with different textures e.g rough, smooth, soft and sponge.

\section{- Exercise 4:}

In completely dark room, therapist used disco lights while supporting the child in standing position on ball. Therapist bounced the child up and down for 2 minutes while encouraging him/ her to follow the movable light spots with eyes and head. Therapist repeated this exercise 3-5 times.

\section{- Exercise 5:}

In completely dark room, therapist used disco lights while encouraging the child to crawl slowly forward and backward, then 
the therapist maintained static position at the end of each range to encourage the child to follow the movable light spots with eyes and head.

- Exercise 6:

While applying disco lights in completely dark room, therapist encouraged the child to walk in circle in the same direction of movable light spots for 2 minutes then, therapist encouraged the child to walk in the opposite direction for another 2 minutes.

\section{B- Traditional physical therapy program:}

The following standing and walking exercises were applied to all children for 60 minutes/ session, 3 times/ week for 3 successive months.

1- Standing on one leg.

2- Standing on balance board.

3- Standing on different textures.

4- Stoops and recovers from standing position.

5- Raising reaction from sitting on chair/ ball to standing position.

6 - Throwing ball from standing position.

7- Kicking ball from standing position.

8- Walking on line.

9- Walking in circle.

10- Walking backward.

11- Walking while using different obstacles e.g stepper, balance board, balance beam, vestibulator, inclined surfaces and so on.

12- Jumping in place.

13- Jumping down using spring.

14- Walking with eyes closed.

\section{Informed consent}

Informed consent has been obtained from all parents of children included in this study.

Statistical analysis

Statistical analyses were conducted using statistical package for the social sciences (SPSS) version 20 for windows. Means and standard deviations of the measured parameters were calculated. Wilcoxon Signed Ranks Test was used to test statistical difference between pre and post treatment measures for the study group. All statistical analyses were equal to or less than 0.05 level of probability.

\section{RESULTS}

\section{(1) Demographic characteristics of study sample}

Twelve children with dyspraxia participated in this study. The mean value of their age was $6.2 \pm 3.06$. The frequency of girls was 3 (25\%), while the frequency of boys was $9(75 \%)$.

(2) Visual function level

Table (1) shows significant improvement in visual function level for the study group when comparing it's results before and after three months of treatment. The mean value before treatment was $2.17 \pm$ 0.57 , while after three months of treatment was $4.25 \pm 0.62$. The mean difference was 2.08 and $Z$ value was 3.213 , which was statistically significance $(P=0.01)$. The percentage of improvement was $105.55 \%$.

(3) Gross motor function

Table (1) shows significant improvement in gross motor function (locomotion subtest of PDMS) for the study group when comparing its results before and after three months of treatment. The mean value before treatment was $1.08 \pm 0.25$, while after three months of treatment was $2.00 \pm 0.95$. The mean difference was 0.92 and $Z$ value was 2.428 , which was statistically significance $(P=0.05)$. The percentage of improvement was $87.5 \%$.

(4) Age equivalent of locomotion

Table (1)shows significant improvement in age equivalent of locomotion for the study group when comparing it's results before and after three months of treatment. The mean value before treatment was $8.58 \pm 2.77$, while after three months of treatment was $12.83 \pm 4.46$. The mean difference was 4.25 and $Z$ value was 3.068 , which was statistically significance $(P=0.05)$. The percentage of improvement was $59.31 \%$.

Table (1): Statistical analysis of visual function, motor function, and age equivalent of locomotion before and after treatment of study sample

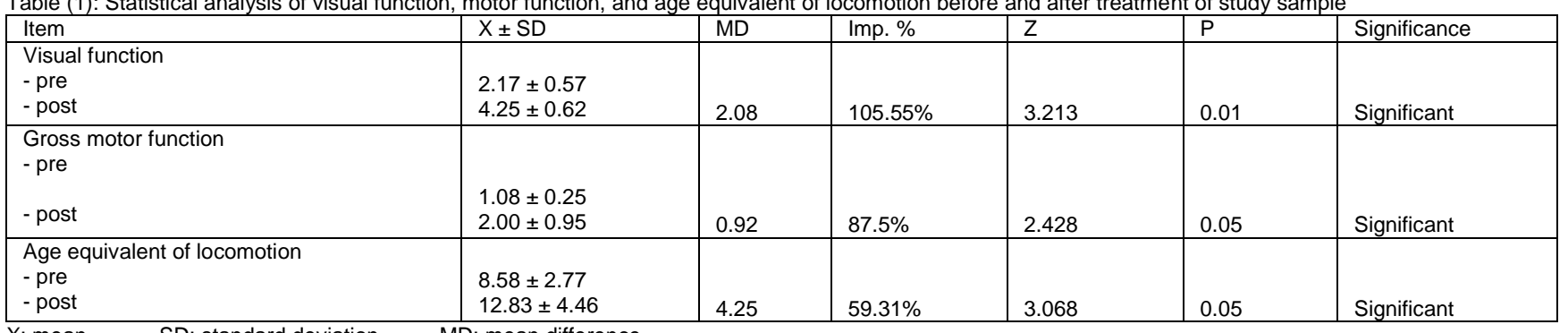

X: mean SD: standard deviation MD: mean difference

Z: willcoxon signed rank value $\quad \mathrm{P}$ : probability value

Imp \%: improvement percent

\section{DISCUSSION}

The purpose of the study was to investigate the effect of vestibular rehabilitation therapy program on the visual and motor functions in children with dyspraxia. Results showed highly significant improvement in visual function level and gross motor functions for the study group when comparing it's results before and after three months of treatment.

The rehabilitation program used in this study was VRT as therapeutic modality used in rehabilitation work for pediatric population. VRT has been defined as an effective modality for most individuals with disorders of the vestibular or central balance system disorders. The basis for the success of VRT is the use of existing neural mechanisms in the human brain for adaptation, plasticity, and compensation. The vestibular system cannot be considered as a separate entity ignoring other balance subsystems. Hence, a modified VRT program, named pediatric balance therapy with special modifications in exercises, was developed for children with vestibular disorders, in accordance to the whole balance system [14]. Also, Melo et al. (2019) ${ }^{[15]}$ stated that Vestibular rehabilitation is a therapeutic resource applied to patients with balance disorders of vestibular origin and its proposal is based on the central mechanisms of neuroplasticity, known as adaptation, habituation and substitution, whose final objective is vestibular compensation. The exercises used in vestibular rehabilitation aim at improving vestibulovisual interaction during cephalic movements, optimizing static and dynamic postural stability under conditions that produce conflicting sensory information. Vestibular rehabilitation has proven to be a safe and effective therapeutic option for treating children with vestibular disorders.

It is intended in this study to select children with dyspraxia aged from 4 to 12 years old. This age is suitable for conducting VRT for those children. This comes in agreement with Lotfi et al. (2016) ${ }^{[14]}$ who developed a modified VRT program for children with vestibular disorders taking into account the whole balance system. The modified system as named as pediatric balance therapy (PBT) with special modifications in exercises to use for children of 4- 12 age group. 
All children in this study had IQ score more than 70 so, they could attend, focus, concentrate, understand orders, give better response to the given stimuli and make a memory for their actions. They had delayed response at first that could be explained by having slow information processing, but by giving them suitable stimuli, offering simple environment, allowing enough time for them to respond, do more and more repetitions and giving harder exercise as the child improved, all these factors helped us in overcoming the information processing problem. This comes in agreement with Montgomery and Connolly (2003) ${ }^{[16]}$ who stated that, cognitive abilities and constraints are important to identify. Cognitive impairments can be a distraction and impede learning and limit balance progressions in treatment. The therapist should consider the patient's cognitive abilities to avoid overtaxing the CNS, resulting in poor performance in physical tasks such as balance. Balance challenge should progress by using more difficult cognitive challenges in combination with balance challenges as appropriate.

Improvement observed in visual level and gross motor activities may be due visual rehabilitation program given in this study which helped children to overcome their visual disability. There was great concentration on the use of visual abilities and functions for rehabilitation of the vestibular system as vision is the first sense that helps the child to identify the world around him through recognizing shapes, colors and sizes of objects. Also, vision allows the child to know about his/ her body parts, the relation of body parts to each other and to the objects in space. Vision gives information about distances in space (spatial orientation). All these information are carried out to the brain aiming for helping the child to make the suitable response to the given stimulus.

Montgomery and Connolly (2003) ${ }^{[16]}$ stated that, the visual system is primarily responsible for receiving information about the environment including the location of objects in space. The visual system also provides proprioceptive information about where our body is in space and the spatial relationship between body parts. In this study, the visual exercises were started in the sensory room using single light source (torch or light toy) in completely dark room to improve gazing, saccadic and pursuit eye movement. Also, children were placed in different positions as supine lying position and sitting whether on ground, chair, balance board or even GYM ball aiming to use motor ability to improve the visual level and to use the gained results for more improvement in motor skills.

Kurtz (2008) [17] reported that children with coordination difficulties often have associated problems with functional vision skills. Functional vision refers to the way the various eye muscles move efficiently to track a moving target. Children with problems affecting bilateral motor integration are particularly susceptible to difficulties with tracking a visual target across their midline, or to accommodating vision to focus on objects at varying distances from the eyes. The results gained in the study group are confirmed by the opinion of Hyvarinen (2004) ${ }^{[18]}$ who stated that the sensory lights for children improve concentration, promote participation and visual stimulation through flashing lights, torches, fiber optics and glow in the dark. The sensory lights for children provide visual challenges, whether a Light Emitting Diode (LED) spinning top or glitter ball. Sensory lights for children provide eye catching fun that inspires visual input and development. Sensory lights for children are ideal for visual processing, improving tracking and increasing eye hand coordination. Persha (2004) [19] assured the benefits of using visual stimulation modalities as she stated that the visual sensory products encourage visual skills of users at many levels of understanding and learning. To encourage visual processing and discrimination, visual perception, visual input, focus, concentration, eye exercise, tracking, color recognition and awareness, increasing hand- eye coordination, spatial vision, encourage success, calm and distract negative behavior, open discussion and encourage participation and social skills, and provide visual challenges.
Improvement in visual function level in study group may be attributed to the use of bright colored lights in completely dark room. By using completely dark room, the surrounding environment around the child became simple so, overcoming the problem of overcrowded vision. Also, the use of dark room offered very quiet environment away from distractive lights to avoid over stimulation and to help in relaxation which leads to improvement of attention. The use of bright colored lights encourages attention and improves gazing, localizing and tracking skills as only one stimulus was given in a very simple environment (the dark room).

Allowing the child to respond well to the giving stimulus by affording him/ her enough time may be also one of the reasons of improvement of visual level in children with dyspraxia who had a delayed response to visual stimulus. Thirty seconds of exposure to light disconnected by five seconds of rest was suitable to the children to give good response to the stimuli. Also, the gradual increase of the time of exposure to the stimulus led to better response. This explanation comes in agreement with palmer (2003) ${ }^{[20]}$ who stated that, in early stages of visual rehabilitation, it may take the child extended periods of time to demonstrate a visual response. Child may demonstrate a delay in using vision to "look at" a target most of the time when a new item is presented or a new activity starts. Allow the child multiple opportunities and extended periods of time to demonstrate a visual response. Latency may decrease as the child uses his/her vision more.

The use of visual motor coordination exercises and proprioceptive training in the present study is supported by opinion of Macintyre and McVitty (2004) ${ }^{[21]}$ who stated that, when the proprioceptive sense is functioning well, the children have a clear idea of their posture and they can make adjustments. The input is processed through the vestibular sense and works with all other sensory sources to aid balance and efficient movement, i.e. with no fluster or clumsiness. It is a complex sense because incorrect information coming from any of the other senses will affect the proprioceptive sense. It can also work to give a sense of position when the visual and auditory senses are not functioning well. Ayres (1980) ${ }^{[12]}$ reported that most children with minimal brain dysfunction show some irregularity in their tactile process. For dyspraxic child, the tactile information is vague and results in a body percept that is not precise. Therefore, in the present study the dyspraxic child was encouraged to direct his/ her own movements. The more he moves himself, the more internal feedback he gives himself. Self-directed movement is one of the keys to developing better motor planning. It is proposed that the carefully controlled sensory input through vestibular and somatosensory systems enhanced the capacity of the brain for intersensory integration between these sensory modalities and the visual and auditory inputs that were a natural component of the gross motor experience employed to elicit much of the stimuli. Mechanisms for these integrative processes exist in the brain stem, thalamus, and cortex. It is believed that considerable processing of visual stimuli occurs in the brain stem and that at that level it is intimately associated with postural and ocular mechanisms. While no overt "eye exercises" in the usual sense were employed, the elicited sensory flow was considered to have a profound and positive influence on extra ocular muscle control.

The exercise program used in the current study was depending mainly on dynamicity rather than stability and use both sides of the body equally for more improvement of body awareness and perception. This made children more confident and had more self-esteem allowing them to participate successfully in the exercise. Thus, the difficulty of exercises was increased by decreasing or eliminating the number of senses the child used. At the beginning of exercise program, the children used vision, proprioception, and vestibular system to adjust their movement. Then, visual input was eliminated as the exercises were performed with eyes closed or in dark room but still using different tactile surfaces to allow proprioceptive stimulation through touch. At the end of the rehabilitation program, children were depending only on the vestibular sense during the exercise. This comes in agreement 
with Lotfi et al. (2016) ${ }^{[14]}$ who confirmed that, while performing the exercises, stress symptoms need to be searched out, such as crying, blushing or fearing. The practice session needs to be ended with the appearance of any of the mentioned symptoms.

The use of proprioceptive sense through tactile stimulation (different tactile surfaces) gives continuous stimulation to the brain from the surrounding environment about where the objects in space and what the body's position is in relation to these objects. Through somatosensory receptors which are located on the body surface, information about texture, temperature and painful stimuli are reached to the brain. Also, standing and walking on different tactile surfaces while using obstacles helped the receptors which are located in the muscles and joints to detect the changes that occurred in the muscle length and tension, joint angles and body's position in space and deliver these changes to the brain.

Montgomery and Connolly (2003) ${ }^{[16]}$ mentioned that, the environment as we know it is created in our brain from information received through our senses. In turn, our behaviors, including motor functions, are produced in relationship to environmental stimuli. These stimuli are perceived, organized, integrated and interpreted by the nervous system to produce an appropriate response, which is often a motor action. A person with a deficit in the nervous system that interferes with interpreting environmental stimuli will likely produce an inefficient movement if asked to perform a motor task. So, the integration of all sensory information is required for planning movements and altering movements already in progress.

In the present study, the target was not only to help the child to reach the desired motor milestones but also, to maintain enough balance and stability whether in static position (standing) or in dynamic pattern e.g walking, crawling and so on. Through integration of the information that gathered from different senses i.e vision, touch, and proprioception, child could maintain sitting position on static surface (ground), dynamic surface (GYM ball), upright posture on even and uneven surfaces, and could walk on different surfaces with/ without support in balanced manner. Also, maintaining balance during movement helped children in this study to make dual task as throwing/ kicking ball while standing.

This comes in agreement with Montgomery and Connolly (2003) ${ }^{[16]}$ who reported that, balance is the ability to maintain the position of the body relative to stability limits. It involves controlling the body's position in space for the purposes of stability and orientation. Orientation is the ability to maintain an appropriate relationship between the body segments and between the body and environment for a task. For most tasks a vertical orientation was maintained using multiple sensory references (eg, gravity, support surface, and relationship of the body to objects in the environment). Stability and orientation are two distinct goals of the postural control system. Most tasks have postural control as a requirement, but the demands of stability and orientation change with each task.

Study limitations: The limitation of this study was small sample size, which does not allow extrapolating the results to the population of children with developmental dyspraxia. Also, the diagnosis dyspraxia is particularly difficult to be established, and children often have co-morbidities. The concept of developmental dyspraxia probably comprises of heterogeneous clinical entities.

\section{CONCLUSION}

It can be concluded that, the use of VRT is a very beneficial program to be used in the rehabilitation of such cases. It is recommended for future research to examine its effect in different pediatric cases with vestibular impairments. Also, it is recommended to apply VRT on a larger sample size.

Acknowledgments: The author thanks the parents of all participants who volunteered for this study for their collaboration.
Disclosure statement: The author does not have any financial interest and did not receive any financial benefit from this research.

Conflict of interest: The author states no conflict of interest.

\section{REFERENCES}

1. Wang T., Tseng M., Wilson B. N. and Hu F.(2009).Functional performance of children with developmental coordination disorder at home and at school. Dev Med Child Neurol. 51 (10): 817- 25.doi: 10.1111/j.1469-8749.2009.03271.x.

2. Gibbs J., Appleton J. and Appleton R..(2007).Dyspraxia or developmental coordination disorder? Arch Dishild.92(6):534-9.doi: 10.1136/adc.2005.088054.

3. Minnis S.(2017). What is dyspraxia?. Dyspraxia: Causes, symptoms, and treatments.

4. Khan S. and Chang R. (2013).Anatomy of the vestibular system: a review. NeuroRehabilitation.32(3): 437- 43.doi: 10.3233/NRE130866.

5. Agrawal Y., Carey J. P., Santina C. C., Schubert M. C., and Minor L. B.(2009).Disorders of balance and vestibular function in US adults: data from the National Health and Nutrition Examination Survey, 2001-2004. Arch Intern Med. 25; 169 (10): 938- 44.doi: 10.1001/archinternmed.2009.66.

6. Rinea R. M. and Wiener-Vacherc S.(2013)Evaluation and treatment of vestibular dysfunction in children. Neuro Rehabilitation 32; 507518. DOI:10.3233/NRE-130873

7. Hain T. C.(2011).Vestibular Rehabilitation Therapy (VRT) for patients who have been referred for vestibular therapy. Chicago dizziness and hearing center.

8. Han B. I., Song H. S. and Kimc J. S.(2011).Vestibular Rehabilitation Therapy: Review of Indications, Mechanisms, and Key Exercises. Journal of Clinical Neurology .7(4):184-196. DOI: 10.3988/jcn.2011.7.4.184

9. Farrell L.(2015).Vestibular Rehabilitation: An Effective, EvidenceBased Treatment. Clinical Faculty, Department of Physical Therapy, Nova Southeastern University, Fort Lauderdale, FL.

10. Cronin G. W (2015).Pediatric Vestibular Rehabilitation: Assessment and Treatment. Pediatric Therapy Associates of South Florida Plantation, FL.

11. Medeiros, Italo R. T; Bittar, Roseli S. M; Pedalini, et al.(2005).Vestibular Rehabilitation Therapy in Children. Otology \& Neurotology, 26(4):699-703. 10.1097/01.mao.0000169051.69254.85

12. Ayres J (1972). Treatment of sensory integrative dysfunction. Australian occupational therapy journal. doi.org/10.1111/j.14401630.1972.tb00547.x

13. Folio MR, Fewell RR. (2000). PDMS-2 Peabody developmental motor scales second edition. Austin: PRO-ED Inc.

14. Lotfi Y., Rezazadeh N., Moossavi A., et al (2016).Review Paper: Introduction of Pediatric Balance Therapy in Children with Vestibular Dysfunction: Review of Indications, Mechanisms, and Key Exercises. Iranian rehabilitation journal. Volume 14 Number 1. http://dx.crossref.org/10.15412/J.IRJ.08140102

15. Melo R. S., Lemos A., Paiva G. S., Ithamar L., Lima M. C., et al (2019).Vestibular rehabilitation exercises programs to improve the postural control, balance and gait of children with sensorineural hearing loss: A systematic review. PubMedhttps://pubmed.ncbi.nlm.nih.gov.Int J Pediatr Otorhinolaryngol127:109650. doi: 10.1016/j.jporl.2019.109650.

16. Montgomery P. and Connolly B. H.(2005). Therapeutic Exercise in Developmental Disabilities. Third edition.ISBN-13: 978-1556426247

17. Kurtz L. A.(2008). Understanding Motor Skills in Children with Dyspraxia, ADHD, Autism, and Other Learning Disabilities: A Guide to Improving Coordination. First edition. ISBN $9781843108108 \mathrm{hb}$

18. Hyvarinen L.(2004). Understanding the behaviors of children with $\mathrm{CVI}$. American printing house for the blind. https://cvi.aphtech.org.

19. Persha A.J.(2004). Visual stimulation activities for infants and toddlers, a guide to parents and caregivers. National institute for the mentally handicapped. Government of India. ISBN 8189001027.

20. Palmer C.(2003). Children with cortical visual impairment: implications for education. School of special education and disability studies. Flinder University.

21. MontgomeryP. C. and Connolly B. H.(2003).Clinical Applications For Motor Control. First edition. ISBN 1-55642-545-7 